\title{
IMAGING DISCOVERY OF THE DEBRIS DISK AROUND HIP 79977*
}

\author{
C. Thalmann ${ }^{1}$, M. Janson ${ }^{2}$, E. Buenzli ${ }^{3}$, T. D. BrandT ${ }^{2}$, J. P. Wisniewsii ${ }^{4}$, C. Dominik ${ }^{1}$, J. Carson $^{5}$, M. W. McElwain $^{6}$, \\ T. Currie $^{7}$, G. R. KnapP ${ }^{2}$, A. Moro-Martín ${ }^{8}$, T. Usuda ${ }^{9}$, L. Abe $^{10}$, W. Brandner ${ }^{11}$, S. Egner $^{9}$, M. Feldi $^{11}$, T. Golota ${ }^{9}$, \\ M. Goto ${ }^{12}$, O. Guyon ${ }^{9}$, J. Hashimoto ${ }^{13}$, Y. Hayano ${ }^{9}$, M. Hayashi ${ }^{9}$, S. Hayashi ${ }^{9}$, T. Henning ${ }^{11}$, K. W. HodapP ${ }^{14}$, M. IshiI ${ }^{9}$, \\ M. IYe ${ }^{13}$, R. KAndori ${ }^{13}$, T. Kudo ${ }^{13}$, N. KusaKabe ${ }^{13}$, M. KuZuhara ${ }^{13,15}$, J. Kwon ${ }^{13,16}$, T. Matsuo ${ }^{13}$, S. MaYAMA ${ }^{16}$, \\ S. Miyama ${ }^{13}$, J.-I. Morino ${ }^{13}$, T. Nishimura ${ }^{9}$, T.-S. Pyo ${ }^{9}$, E. Serabyn ${ }^{17}$, H. Suto ${ }^{13}$, R. Suzuki ${ }^{13}$, M. Takami ${ }^{18}$, N. Takato ${ }^{9}$, \\ H. Terada ${ }^{9}$, D. Tomono ${ }^{9}$, E. L. Turner ${ }^{2,19}$, M. Watanabe ${ }^{20}$, T. YAmada $^{21}$, H. TAKAmi $^{9}$, And M. TAmura ${ }^{13}$ \\ ${ }^{1}$ Astronomical Institute "Anton Pannekoek," University of Amsterdam, Amsterdam, The Netherlands; thalmann@ uva.nl \\ ${ }^{2}$ Department of Astrophysical Sciences, Princeton University, Princeton, NJ, USA \\ ${ }^{3}$ Department of Astronomy and Steward Observatory, University of Arizona, Tucson, AZ, USA \\ ${ }^{4}$ H.L. Dodge Department of Physics \& Astronomy, University of Oklahoma, OK, USA \\ ${ }^{5}$ Department of Physics and Astronomy, College of Charleston, Charleston, SC, USA \\ ${ }^{6}$ NASA Goddard Space Flight Center, Greenbelt, MD, USA \\ ${ }^{7}$ Department of Astronomy and Astrophysics, University of Toronto, Toronto, Canada \\ ${ }^{8}$ Department of Astrophysics, CAB-CSIC/INTA, Madrid, Spain \\ ${ }^{9}$ Subaru Telescope, Hilo, HI, USA \\ ${ }^{10}$ Laboratoire Hippolyte Fizeau, Nice, France \\ ${ }^{11}$ Max Planck Institute for Astronomy, Heidelberg, Germany \\ 12 Universitätssternwerte München, Ludwig-Maximilians-Universität, Munich, Germany \\ ${ }^{13}$ National Astronomical Observatory of Japan, Tokyo, Japan \\ ${ }^{14}$ Institute for Astronomy, University of Hawai 'i, Hilo, HI, USA \\ ${ }^{15}$ Department of Earth and Planetary Science, University of Tokyo, Tokyo, Japan \\ ${ }^{16}$ Department of Astronomical Science, Graduate University for Advanced Studies (Sokendai), Shonan Village, Japan \\ ${ }^{17}$ NASA Jet Propulsion Laboratory, California Institute of Technology, Pasadena, CA, USA \\ ${ }^{18}$ Institute of Astronomy and Astrophysics, Academia Sinica, Taipei, Taiwan \\ ${ }^{19}$ Kavli Institute for the Physics and Mathematics of the Universe, University of Tokyo, Kashiwa, Japan \\ ${ }^{20}$ Department of Cosmosciences, Hokkaido University, Sapporo, Japan \\ ${ }^{21}$ Astronomical Institute, Tohoku University, Sendai, Japan \\ Received 2012 October 16; accepted 2012 December 20; published 2013 January 14
}

\begin{abstract}
We present Subaru/HiCIAO $H$-band high-contrast images of the debris disk around HIP 79977, whose presence was recently inferred from an infrared excess. Our images resolve the disk for the first time, allowing characterization of its shape, size, and dust grain properties. We use angular differential imaging (ADI) to reveal the disk geometry in unpolarized light out to a radius of $\sim 2^{\prime \prime}$, as well as polarized differential imaging to measure the degree of scattering polarization out to $\sim 1^{\prime \prime} .5$. In order to strike a favorable balance between suppression of the stellar halo and conservation of disk flux, we explore the application of principal component analysis to both ADI and reference star subtraction. This allows accurate forward modeling of the effects of data reduction on simulated disk images, and thus direct comparison with the imaged disk. The resulting best-fit values and well-fitting intervals for the model parameters are a surface brightness power-law slope of $S_{\text {out }}=-3.2[-3.6,-2.9]$, an inclination of $i=84^{\circ}\left[81^{\circ}, 86^{\circ}\right]$, a high Henyey-Greenstein forward-scattering parameter of $g=0.45[0.35,0.60]$, and a nonsignificant disk-star offset of $u=3.0[-1.5,7.5] \mathrm{AU}=24[-13,61]$ mas along the line of nodes. Furthermore, the tangential linear polarization along the disk rises from $\sim 10 \%$ at $0^{\prime \prime} .5$ to $\sim 45 \%$ at $1^{\prime \prime} .5$. These measurements paint a consistent picture of a disk of dust grains produced by collisional cascades and blown out to larger radii by stellar radiation pressure.
\end{abstract}

Key words: circumstellar matter - planetary systems - stars: individual (HIP 79977) - techniques: high angular resolution

Online-only material: color figures

\section{INTRODUCTION}

Most debris disk systems are second-generation disks produced by the collisional destruction of planetesimals, although some of the youngest systems $(\sim 10 \mathrm{Myr})$ might also include primordial star formation material (e.g., Wyatt 2008). While space-based infrared (IR) surveys have identified many debris disks (e.g., Aumann et al. 1984), though few have been spatially resolved at optical or IR wavelengths. High-resolution images

\footnotetext{
* Based on data collected at Subaru Telescope, which is operated by the National Astronomical Observatory of Japan.
}

have revealed morphological disk sub-structures such as warps, geometrical offsets, and clumps (e.g., Kalas \& Jewitt 1995; Heap et al. 2000; Kalas et al. 2005; Lagrange et al. 2009), possibly caused by gravitational perturbations from exoplanets. In particular, identifying new debris disk systems with an edge-on inclination is critical to efforts to study the origin and composition of their small, second-generation gaseous components (Roberge et al. 2006).

HIP 79977 (HD 146897) is a 5-10 Myr old (de Zeeuw et al. 1999; Pecaut et al. 2012; Song et al. 2012) F2/3V member of Upper Scorpius at a distance of $123_{-14}^{+18}$ pc (van Leeuwen 2007) which has been identified as a debris disk system with a 
fractional IR luminosity $L_{\mathrm{IR}} / L_{\star}=5.9 \times 10^{-3}$ (Chen et al. 2006, 2011). A simple single-temperature fit to its IR excess led Chen et al. (2011) to suggest a source ring radius of at least $40 \mathrm{AU}$, with a characteristic temperature of $89 \mathrm{~K}$. Here we present highcontrast imaging of HIP 79977 which resolves this debris disk in scattered light for the first time.

\section{OBSERVATIONS}

As part of the SEEDS survey (Strategic Exploration of Exoplanets and Disks with Subaru/HiCIAO; Tamura 2009), we obtained two epochs of high-contrast imaging on HIP 79977 with the HiCIAO instrument (Hodapp et al. 2008) on the Subaru Telescope.

The first data set was taken in $H$ band $(1.6 \mu \mathrm{m})$ on 2012 May 12, comprising 119 frames of $20 \mathrm{~s}$ for a total exposure time of 39.7 minutes, and spanning 19.6 of field rotation. The image rotator was operated in pupil-tracking mode to enable angular differential imaging (ADI; Marois et al. 2006). The plate scale was $9.50 \pm 0.02$ mas pixel $^{-1}$, and the field of view $20^{\prime \prime} \times 20^{\prime \prime}$. The AO188 adaptive optics system (Minowa et al. 2010) provided a stable point-spread function (PSF) with an FWHM of 6.7 pixels $=64$ mas under good weather conditions ( 0'.6 DIMM seeing). HIP 79977 saturated out to a radius of 22 pixels $=0$ '. 2 ; no coronagraph was used. After correction for flat-field and field distortion (Suzuki et al. 2010), the images were registered by fitting a Moffat profile to the PSF halo, for an estimated registration accuracy of 0.5 pixels $=5$ mas.

The second data set was taken in two-channel polarized differential imaging (PDI) mode on 2012 July 7 under the same filter, plate scale, and saturation conditions, with excellent seeing (0.'4). It comprises 64 frames of $30 \mathrm{~s}$ for a total exposure time of 32 minutes, and spans 17.3 of field rotation. The field of view was $10^{\prime \prime} \times 20^{\prime \prime}$. The exposures are organized into serial batches of four frames in which HiCIAO's half-wave plate cycled through the position angles $\left(0^{\circ}, 45^{\circ}, 22.5,67.5\right)$, measuring the Stokes linear polarization parameters $(I+Q$, $I-Q, I+U, I-U)$, respectively. This allows for correction of non-common path aberrations by double-difference polarimetry on short timescales. Each frame was registered by Moffat fitting. While the intensity component of the PDI data set can also be reduced with $\mathrm{ADI}$, the resulting quality is inferior to that of the dedicated 2012 May ADI data. We therefore use the 2012 May data to constrain the full-intensity appearance of the HIP 79977 disk, and the 2012 July data to obtain the matching polarizedintensity image.

\section{DATA REDUCTION}

\subsection{Angular Differential Imaging}

While the ADI technique with the LOCI algorithm (Lafrenière et al. 2007) is commonly used to improve highcontrast sensitivity for detecting extrasolar planets (e.g., Marois et al. 2010; Lagrange et al. 2010) and brown dwarf companions (e.g., Thalmann et al. 2009), we recently demonstrated its use for detecting faint circumstellar disks hidden in the speckle halo of their host stars (Thalmann et al. 2010, 2011; Buenzli et al. 2010). This "conservative LOCI" technique has since been widely adopted (Rodigas et al. 2012; Currie et al. 2012; Boccaletti et al. 2012; Lagrange et al. 2012).

The benefit of powerful speckle suppression with ADI comes at the price of partial loss of disk flux and morphological integrity (Milli et al. 2012). However, careful forward modeling of these effects can recover the physical disk morphology, as we demonstrated on the HR 4796 A debris disk (Thalmann et al. 2011). Clarification is needed regarding recent discussions that attributed some results based on this technique to ADI artifacts rather than to astrophysical processes (Lagrange et al. 2012; Milli et al. 2012). Since ADI works in concentric annuli, it cannot generate spurious signals at a radius where no disk flux is present. The presence of "streamer"-like flux distributions therefore does imply the presence of physical emission at those radii, even though the streamer morphology itself is known to be carved from a physically smooth disk by the ADI process. The conclusions in Thalmann et al. (2011) therefore remain valid, and we continue to use this methodology in this work.

For HIP 79977, we started with classical ADI (no frame selection; Marois et al. 2006) and conservative LOCI $\left(N_{\delta}=\right.$ $\left.0.75, N_{\mathrm{A}}=10,000\right)$. Furthermore, we adopted the application of principal component analysis (PCA) to ADI as a robust and deterministic alternative to LOCI (Soummer et al. 2012). Closely following the recipe of Amara \& Quanz (2012), we performed PCA on the stack of centered, pupil-stabilized frames after subtracting the mean image and masking the saturated central region $(r \leqslant 22$ pixels $=0.2)$. We then projected each frame onto the first $n$ modes of the orthonormal set of Karhunen-Loève eigenimages delivered by PCA, and subtracted those projections from the frame. The stack of frames was then derotated and collapsed to yield the final image. We hereafter refer to this method as PCA-ADI. Through visual inspection of the resulting images, we found that $n=5$ provides an excellent trade-off between suppression of the stellar PSF halo and conservation of disk flux. Note that PCA-ADI with $n=0$ is identical to classical ADI. To ensure linearity of our data reduction process, we use mean-based rather than medianbased frame combination in all techniques (cf. Brandt et al. 2012).

Finally, we explored the use of PCA for PSF subtraction with reference stars. However, we found that for HiCIAO $H$-band data, the speckle halo varied too much between the science and the reference targets to reach competitive contrasts.

Scattered light from the debris disk around HIP 79977 is clearly detected in the 2012 May data set with all ADIbased techniques, as shown in Figures 1(a)-(c). The ripple pattern visible in the first two images is a spurious residual of Subaru's spider diffraction pattern. Only the PCA-ADI technique proves effective at removing this artifact and revealing the smooth slope of the debris disk. We therefore choose it as the benchmark technique for further analysis, as discussed in Section 4. Figure 1(d) shows the signal-to-noise $(\mathrm{S} / \mathrm{N})$ map of the PCA-ADI image, calculated as the standard deviation in concentric annuli after smoothing with a 5 pixel diameter circular aperture. A box containing the disk flux was masked for noise calculation.

The disk presents itself almost edge-on, and thus appears as a ribbon in the ADI images. Most of the flux is concentrated to the south of the projected disks' major axis, identifying this as the disk's likely near side and indicating strong anisotropic forward scattering. The disk flux remains statistically significant from $\sim 0^{\prime} .3(\approx 40 \mathrm{AU})$ out to $\sim 2^{\prime \prime}$ ( $\approx 250 \mathrm{AU}$ in projection).

\subsection{Polarized Differential Imaging}

We reduced the 2012 July PDI data using the standard procedure for SEEDS polarimetry (cf. Tanii et al. 2012). It includes double-difference polarimetry using four half-wave plate position angles, as well as modeling and correction of 


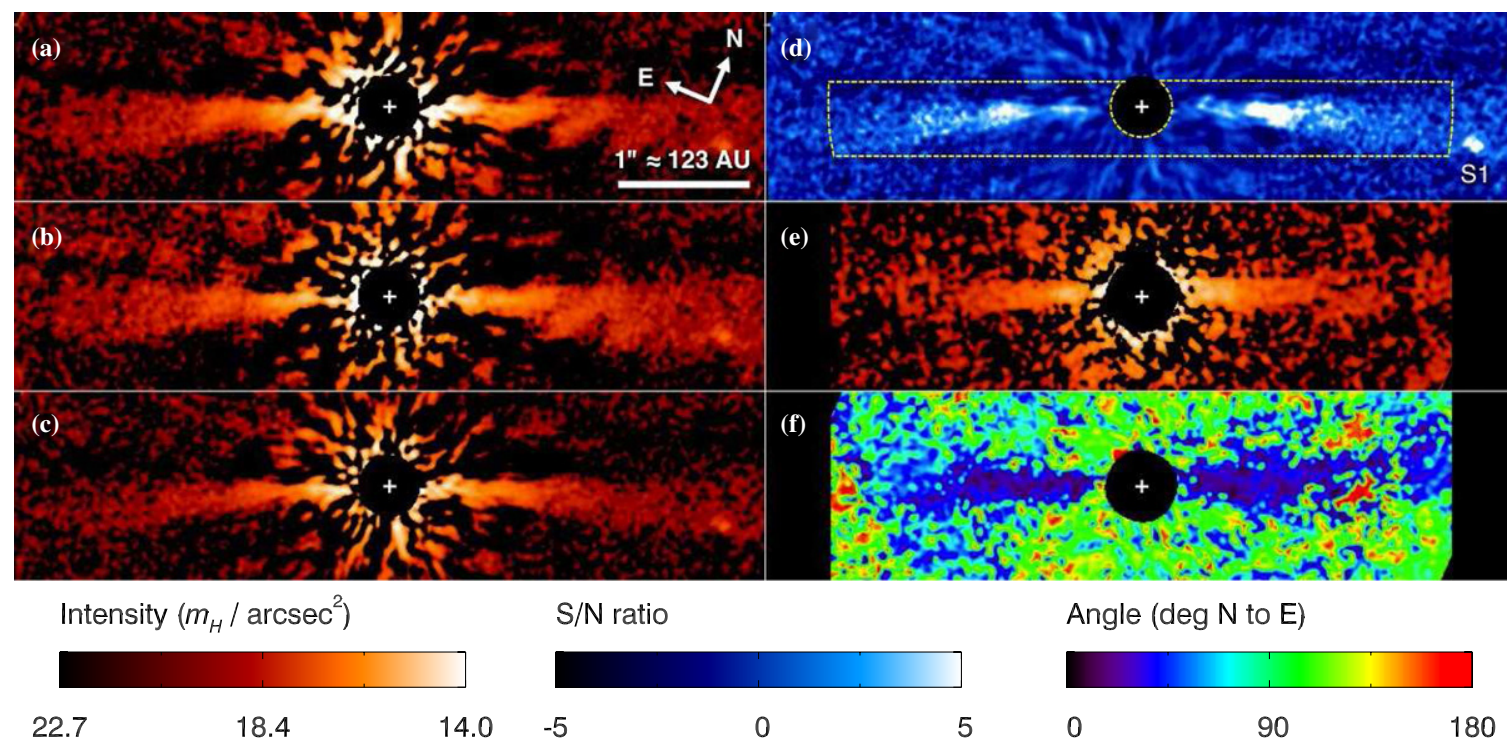

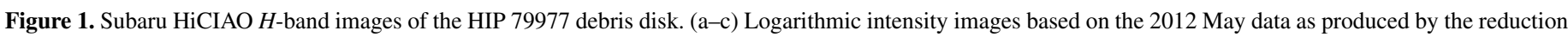

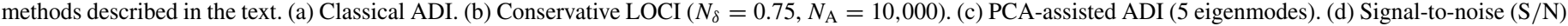

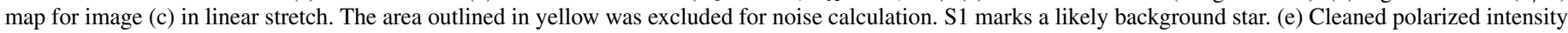

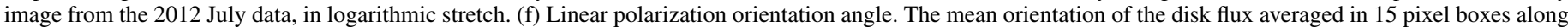

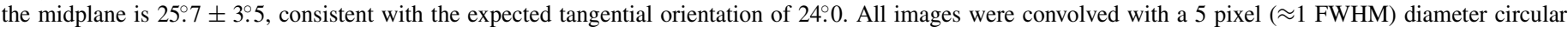
aperture.

(A color version of this figure is available in the online journal.)

instrumental polarization based on Joos et al. (2008). The intensity PSF was fit to the $Q$ and $U$ images and subtracted to remove contributions from a polarized stellar PSF. The resulting polarized intensity image $P=\sqrt{\left(Q^{2}+U^{2}\right)}$ was found to contain spurious structures left behind by the subtraction of imperfectly aligned PSFs, which we removed by projecting spatial derivatives of the intensity PSF to the $Q$ and $U$ images, and subtracting them. Finally, we noticed that rms of the positive and negative residual speckle noise in the $Q$ and $U$ images added up to a positive radial halo in the $P$ image. We measured this noise profile at position angles that do not show disk flux, and subtracted it in quadrature from the entire image.

The resulting $P$ image shows the debris disk out to a separation of $r \approx 1^{\prime \prime} .5$, where the polarized flux level vanishes into the background (Figure 1(e)). The polarization vectors are tangentially oriented, consistent with scattering polarization (Figure 1(f)). No brightness contrast between the near and far side of the disk is evident, in accordance with the expectation that the excess scattered light from forward scattering on large grains is unpolarized.

\section{ANALYSIS}

\subsection{Disk Modeling}

In order to extract physical disk information from our PCAADI image, we generate simulated scattered-light images of model disks using the GRaTer code (Augereau et al. 1999). The main free parameters of the model are the semimajor axis of the source ring $a_{0}$, the inner and outer power-law slopes $\alpha_{\text {in }}, \alpha_{\text {out }}$ of the radial density distribution, the disk-star offset $u$, the inclination angle $i$, the position angle $\phi$, and the Henyey-Greenstein parameter $g$ characterizing the anisotropy of the scattering behavior of the dust grains. For definitions, we refer to Augereau et al. (1999). Note that the measurable outer slope of the disks radial surface brightness profile is not $\alpha_{\text {out }}$, but $S_{\text {out }}=\alpha_{\text {out }}+\beta-2$, where $\beta$ represents the radial shape of the scale height distribution. While this relationship breaks down for purely edge-on disks, it holds for the radial profile for our models with $i \leqslant 88^{\circ}$. We fix $\beta=1$ and henceforth use $S_{\text {out }}$ to characterize the disks outer slope. Since we do not resolve the gap inside the source ring, we adopt the semimajor axis $a_{0}=40 \mathrm{AU}\left(\approx 0^{\prime \prime} 3\right)$ derived from infrared excess by Chen et al. (2011).

As a first step, we determine the position angle $\phi$ of the projected disk's axis of symmetry. We rotate the PCA-ADI image from its north-up, east-left orientation by a given angle, mirror this image about the $y$-axis, and subtract it from an unmirrored copy, adjusting the angle to minimize the subtraction residuals. To quantify the residuals, we first bin the residual image by a factor of $5(\approx 1$ FWHM) in both dimensions, and define an evaluation region of $N_{\text {data }}=1025$ binned pixels framing the disk flux (Figure 2). We derive a noise profile as the standard deviation of pixel values in concentric annuli in the residual image, excluding the evaluation region. We then divide the residual image by this noise profile and calculate $\chi^{2}$ as the sum of squares of all pixel values in the evaluation region.

Our data reduction does not yield a residual map in which the pixels have fully independent normal errors, even after binning. Therefore, we do not expect the usual $\chi^{2}$ thresholds to produce reliable confidence intervals, and use a much more conservative $\chi^{2}$ threshold of $\sqrt{2 N_{\text {data }}}$. Rather than determining confidence intervals about a best-fit model, we seek to determine the family of models consistent with our images.

For the position angles, this yields best-fit value of $\phi=$ 24.0 (counterclockwise from north) and a well-fitting range of [23:7, 24.3]. This includes HiCIAO's True North offset of $0.35 \pm 0.02$ calibrated using van der Marel et al. (2007).

Next, we attempt to match the observed disk morphology by calculating a grid of model disks with $i=\left[77^{\circ} \ldots 88^{\circ}\right]$, $S_{\text {out }}=[-2.8 \ldots-3.7]$, and $g=[0.00 \ldots 0.80]$. Rather than implanting the models into the raw data as we did in Thalmann et al. (2011), we exploit the deterministic nature of PCA-ADI to 


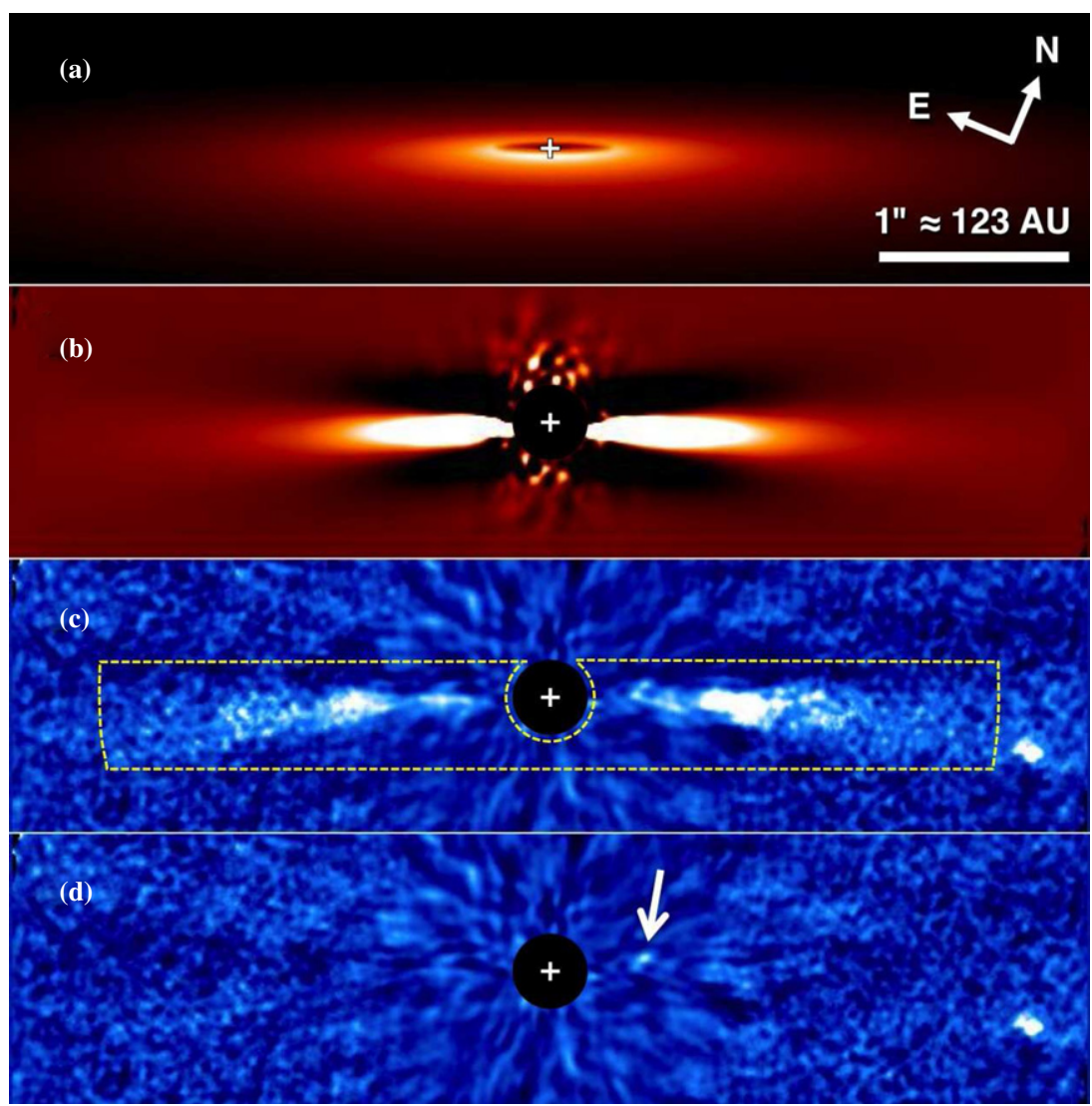

Figure 2. Modeling of the HIP 79977 debris disk. (a) Simulated logarithmic scattered-light image of the best-fit model disk. (b) The same after applying PCA-ADI data reduction (linear stretch to show oversubtraction). (c) Noise-normalized residual map of the PCA-ADI reduction of the 2012 May data, at a linear stretch of $[-2.5 \sigma, 5 \sigma]$. The evaluation region is marked with a yellow outline. (d) The same calculated after subtraction of the model disk treated with PCA-ADI. The disk flux is effectively removed from the data (residual $\chi_{\min }^{2}=1095=1.10 N_{\text {data }}$ ). An unconfirmed point-like signal at $4.6 \sigma$ significance is highlighted with an arrow. For visual clarity, no binning has been applied for panels (c) and (d).

(A color version of this figure is available in the online journal.)

Table 1

Summary of Properties and Results for HIP 79977

\begin{tabular}{|c|c|c|}
\hline Fixed Disk Model Parameters & Adopted & Constraints \\
\hline Source ring semimajor axis $a_{0}\left(\mathrm{AU},{ }^{\prime \prime}\right)^{\mathrm{a}}$ & $40 \approx 0 ! 33$ & $<60 \approx 0.49$ \\
\hline Inner brightness distribution slope $\alpha_{\text {in }}$ & 20 & \\
\hline Scale height radial shape parameter $\beta$ & 1 & \\
\hline Optimized disk model parameters & best fit & well-fitting range \\
\hline Minor axis position angle $\phi\left(^{\circ}\right)$ & 24.0 & {$[23.7,24.3]$} \\
\hline Inclination $i\left(^{\circ}\right)$ & 84 & {$[81,86]$} \\
\hline Henyey-Greenstein parameter $g$ & 0.45 & {$[0.35,0.60]$} \\
\hline Disk-star offset $u(\mathrm{AU})^{\mathrm{b}}$ & 3.0 & {$[-1.5,+7.5]$} \\
\hline Eccentricity $e=\operatorname{abs}\left(u / a_{0}\right)$ & 0.06 & {$[0,0.16]$} \\
\hline \multicolumn{3}{|c|}{ Outer brightness distribution slope $S_{\text {out }}=\alpha_{\text {out }}+\beta-2$ : } \\
\hline model disk, after PCA-ADI & -2.6 & {$[-2.9,-2.3]$} \\
\hline measured, after PCA-ADI & -2.7 & {$[-2.8,-2.6]$} \\
\hline Disk/star flux contrast, $H$ band & 0.0033 & {$[0.0029,0.0038]$} \\
\hline Degree of scattering polarization & Measured & $1 \sigma$ interval \\
\hline At $0.5 \approx 62 \mathrm{AU}(\%)$ & 10 & {$[5,20]$} \\
\hline At $1^{\prime \prime} 0 \approx 123 \mathrm{AU}(\%)$ & 35 & {$[20,45]$} \\
\hline At $1^{\prime \prime} .5 \approx 185 \mathrm{AU}(\%)$ & 45 & {$[30,60]$} \\
\hline
\end{tabular}

Notes. The given ranges for parameters $i, g, S_{\text {out }}, e$, and $u$ represent the minimum and maximum values of the well-fitting family of disk models defined by $\chi^{2} \leqslant \chi_{\min }^{2}+\sqrt{2 N_{\text {data }}}$; see the text and Figure 2 for details.

${ }^{\text {a }} \mathrm{S} / \mathrm{N}$ ratios near the inner working angle are too low for optimization of $r_{0}$; we adopt the value of $40 \mathrm{AU}$ from Chen et al. (2011).

$\mathrm{b}$ The eccentric disk is approximated as a symmetric disk offset from the star along the line of nodes. Positive values of $u$ indicate a disk center to the west of the star. 


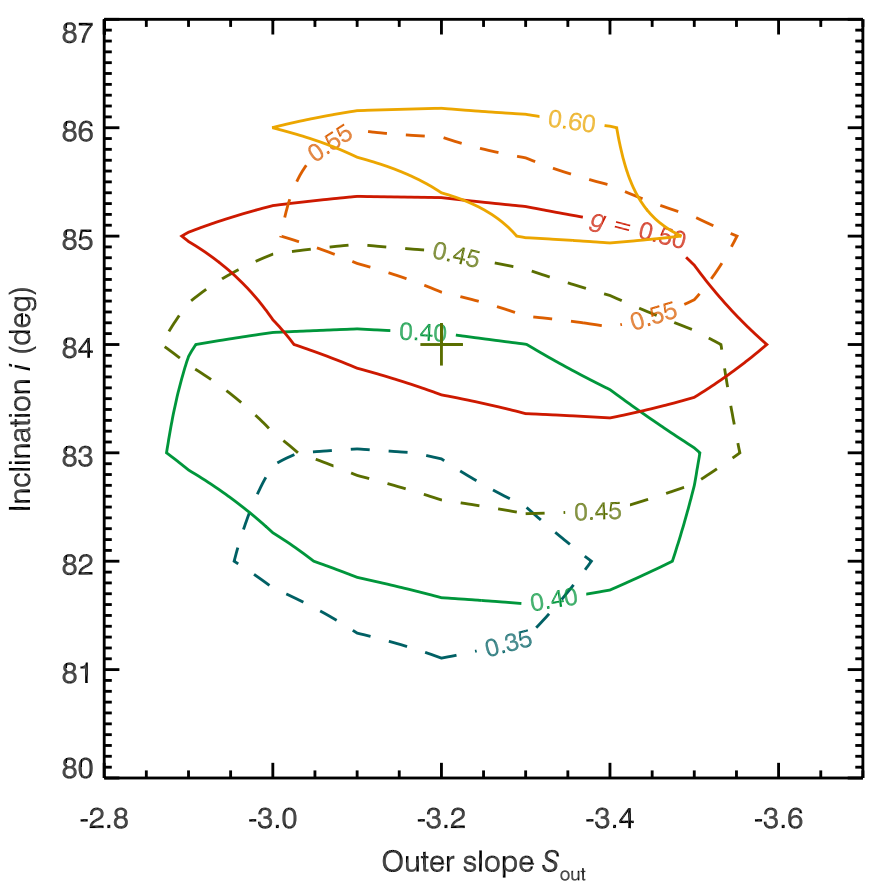

Figure 3. Constraints on the disk model parameters $S_{\text {out }}$ (outer slope), $i$ (inclination), and $g$ (Henyey-Greenstein parameter). The contours delimit the parameter space of well-fitting solutions $\left(\chi^{2} \leqslant \chi_{\min }^{2}+\sqrt{2 N_{\text {data }}}\right)$ for fixed values of $g$. The best-fit solution $\left(\chi^{2}=\chi_{\min }^{2}\right)$ lies in the $g=0.45$ plane; it is marked with a plus sign.

(A color version of this figure is available in the online journal.)

forward model the exact effect of our data reduction process on the model disk (cf. Soummer et al. 2012). We subtract a scaled version of this processed disk image from the data, choosing the scale factor to minimize residual $\chi^{2}$ as defined above. This yields best-fit model parameters and well-fitting parameter ranges of $i=84^{\circ}\left[81^{\circ}, 86^{\circ}\right], S_{\text {out }}=-3.2[-3.6,-2.9], g=0.45$ $[0.35,0.60]$, and a ratio of total disk flux to stellar flux of $3.3[2.9,3.8] \times 10^{-3}$. The minimum $\chi^{2}$ achieved is $1142=$ $1.1 N_{\text {data }}$. The well-fitting family is characterized graphically in Figure 3 and summarized in Table 1. The parameters $i$ and $g$ are somewhat degenerate; higher inclinations mimic enhanced forward scattering.

In a final step, we explore small non-zero eccentricities of the disk while keeping the best-fit values of $i, S_{\text {out }}$, and $g$ fixed. We approximate eccentric model disks by translating a circularly symmetric disk by an offset $u$ along the line of nodes (roughly corresponding to the projected disk's major axis), for an eccentricity of $e=u / a$. Positive values of $u$ are assigned to offsets to the west. We find a best-fit offset of $u=+2.4 \mathrm{AU}=20$ mas, with a well-fitting interval of $[-1.5,+6.3]$ AU. While the best-fit offset corresponds to an eccentricity of $e=0.06$, the well-fitting range is consistent with zero eccentricity.

Figure 2 illustrates this best-fit model and its subtraction residual. The model fully explains the observed image morphology down to the noise threshold.

\subsection{Surface Brightness Profiles}

As a complementary approach, we directly measure the surface brightness profile of the debris disk. To this end, we convolve the PCA-ADI image with a 5 pixel diameter circular aperture and average azimuthally in radial sectors of $9^{\circ}$ centered on the position angles of the traces of the imaged disk. The results are shown in Figure 4(a). The profiles are well described by a power law with a slope of $S_{\text {data }}=-2.7 \pm 0.1$.

While the best-fit model disk found in Section 4.1 has an intrinsic surface brightness slope of $S_{\text {out }}=-3.2$, the forward modeling of the effects of PCA-ADI data reduction on that disk yields a softened slope of $S=-2.6 \pm 0.3$, consistent with our data.

\subsection{Linear Polarization}

We measure the polarized intensity profile in sectors of $6^{\circ}$ centered on the disk traces in the fully reduced $P$ image, from which the rms halo from residual speckle noise has been subtracted. We confirm that the linear polarization is oriented tangentially with respect to the star (Figure 1(f)), as is expected for scattering polarization. To calculate the degree of scattering polarization, we divide the polarized intensity profile by the full intensity profile of the best-fit model disk evaluated in the same sectors.

The results are presented in Figure 4(b). We find that the scattering polarization increases from $\sim 10 \%$ at 0 . 5 to $\sim 45 \%$ at 1 ..5. This might be due to scattering angles at large separations for the considered position angles being constrained to $\sim 90^{\circ}$, where scattering produces the highest amount of polarization. Close to the star, anisotropic forward scattering at angles $>90^{\circ}$ yields a surplus of unpolarized light, diluting the degree of polarization.

\subsection{Point Sources}

Two faint point sources are clearly visible within $3^{\prime \prime}$ of HIP 79977 in the ADI data, one of which is shown in Figure 1(d). A preliminary astrometric analysis based on marginal detections in archival Gemini NICI data identifies both as likely unrelated background stars, though more precise astrometry is needed for a decisive result. Furthermore, subtraction of the best-fit model disk leaves behind a point-like signal of $4.6 \sigma$ significance at a separation of 0.'5 (cf. Figure 2(d)). If confirmed, such a signal may represent scattered light from a localized clump in the debris disk, or the thermal emission from a young planet of 3-5 Jupiter masses (based on Baraffe et al. (2003) and partial self-subtraction correction). While we detect no warps or gaps in the disk, this does not preclude the presence of a planet of the proposed mass and separation range orbiting outside the disk's source ring (Thébault et al. 2012).

\section{DISCUSSION}

The results of our imaging characterization of the debris disk around HIP 79977 fit very well with the emerging standard picture of debris disks, where dust is produced in a ring of colliding planetesimals and then distributed inward and outward by radiation forces. The slope of the scattered-light surface brightness is measured to be within $[-3.6,-2.9]$, which is consistent with the theoretical value of -3.5 typical for a radiation pressure driven eccentricity distribution of particles near blow-out size (Krivov et al. 2006; Strubbe \& Chiang 2006; Thébault \& Augereau 2007). No significant eccentricity of the disk as a whole is measured, potentially setting HIP 79977 apart from other disks like those around Fomalhaut (Chiang et al. 2009; Acke et al. 2012) and HR 4796 A (Thalmann et al. 2011). However, future observations of HIP 79977 resolving the source ring may reveal asymmetries below the current uncertainty threshold of $e \leqslant 0.16$. 

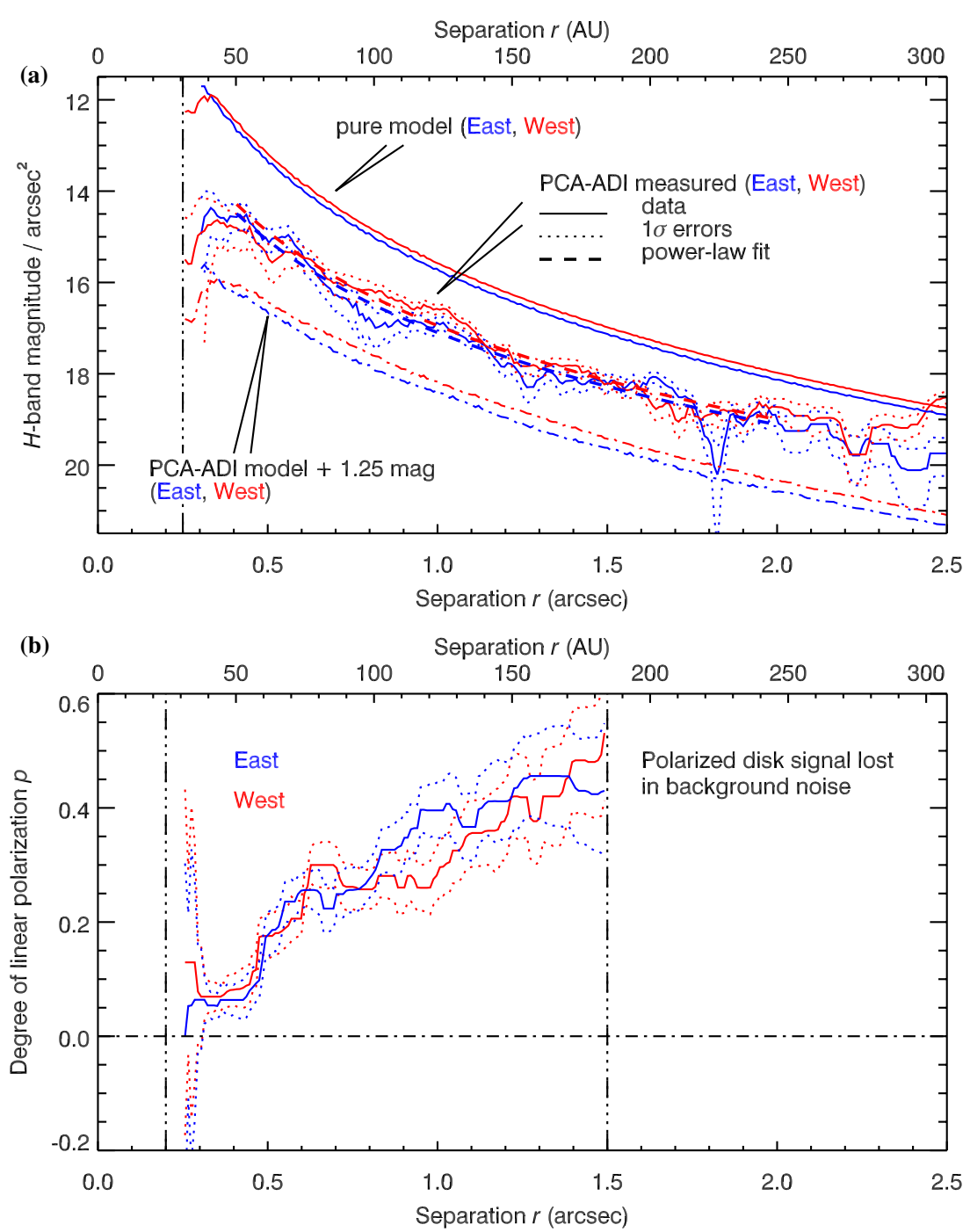

Figure 4. Intensity and polarization profiles of the HIP 79977 debris disk. (a) Surface brightness profiles measured in wedges of $9^{\circ}$ along the eastern and western traces of the debris disk image between position angles $\left[113^{\circ}, 122^{\circ}\right]$ and $\left[286^{\circ}, 295^{\circ}\right]$, respectively. The image was smoothed with a 5 pixel diameter circular aperture prior to extraction. The solid curves with dotted error sheaths represent the profiles measured on the 2012 May data processed with PCA-ADI. The errors were obtained from evaluating a number of $9^{\circ}$ wedges placed in the background at disk-free position angles. Power-law fits to those profiles are overplotted as dashed curves. The fitted slopes are $-2.6 \pm 0.1$ on both sides of the disk. Furthermore, the surface brightness profiles of the best-fit model disk before (top solid curves) and after PCA-ADI treatment (bottom dash-dotted curves) are shown for comparison. The latter are offset downward by $+1.25 \mathrm{mag} \mathrm{arcsec}^{-2}$ to avoid blending with the profiles measured from the data. The pure model profiles are not artificially offset; the difference of 1-2.5 mag $\operatorname{arcsec}^{-2}$ with respect to the measured disk profile represents the flux loss incurred in PCA-ADI. (b) Degree of scattering polarization $p$ at a function of separation, calculated from the polarized intensity image $(P)$ measured in the 2012 July data and the full intensity $I$ image of the best-fit disk model derived from the 2012 May data. The profiles are measured in $6^{\circ}$ wedges and smoothed with a median filter of 11 pixels $(\approx 2 \mathrm{FWHM})$. The polarized disk flux is lost in the background noise beyond 1".5 (cf. Figure 1(e)).

(A color version of this figure is available in the online journal.)

The derived Henyey-Greenstein parameter of $g \approx 0.45$ is consistent with measurements of cometary dust (Kolokolova et al. 2004). Likewise, a maximum degree of polarization of $45 \pm 15 \%$ in $H$ band agrees well with an extrapolation to higher phase angles of observations of high-polarization comets like comet Hale-Bopp (Hasegawa et al. 1997).

As a caveat, we note that other well-fitting disk architectures may exist outside the limitations of our model assumptions, and that the possible degeneracies of PCA-ADI images of disks have not been investigated in depth.

We thank Jean-Charles Augereau for his GRaTer code, and the anonymous referee for helpful comments. J.C. is supported by the US National Science Foundation under Award No. 1009203. The authors recognize and acknowledge the significant cultural role and reverence that the summit of Mauna Kea has always had within the indigenous Hawaiian community. We are most fortunate to have the opportunity to conduct observations from this mountain.

Facility: Subaru (HiCIAO, AO188)

\section{REFERENCES}

Acke, B., Min, M., Dominik, C., et al. 2012, A\&A, 540, A125

Amara, A., \& Quanz, S. 2012, MNRAS, 427, 948

Augereau, J. C., Lagrange, A. M., Mouillet, D., Papaloizou, J. C. B., \& Grorod, P. A. 1999 , A\&A, 348, 557

Aumann, H. H., Beichman, C. A., Gillett, F. C., et al. 1984, ApJL, 278, 23

Baraffe, I., Chabrier, G., Barman, T. S., Allard, F., \& Hauschildt, P. H. 2003, A\&A, 402, 701

Boccaletti, A., Augereau, J.-C., Lagrange, A.-M., et al. 2012, A\&A, 544, A85 
Brandt, T. D., McElwain, M. W., Turner, E. L., et al. 2012, arXiv:1209.3014 Buenzli, E., Thalmann, C., Vigan, A., et al. 2010, A\&A, 524, L1

Chen, C. H., Mamajek, E. E., Bitner, M. A., et al. 2011, ApJ, 738,122

Chen, C. H., Sargent, B. A., Bohac, C., et al. 2006, ApJS, 166, 351

Chiang, E., Kite, E., Kalas, P., Graham, J. R., \& Clampin, M. 2009, ApJ, 693,734

Currie, T., Rodigas, T. J., Debes, J., et al. 2012, ApJ, 757, 28

de Zeeuw, P. T., Hoogerwerf, R., de Bruijne, J. H. J., Brown, A. G. A., \& Blaauw, A. 1999, AJ, 117, 354

Hasegawa, H., Ichikawa, T., Abe, S., et al. 1997, EM\&P, 78, 353

Heap, S. R. 2000, ApJ, 539, 435

Hodapp, K. W., Suzuki, R., Tamura, M., et al. 2008, Proc. SPIE, 7014, 42

Joos, F., Buenzli, E., Schmid, H. M., \& Thalmann, C. 2008, Proc. SPIE, 7016, 70161I

Kalas, P., Graham, J. R., \& Clampin, M. 2005, Natur, 435, 1067

Kalas, P., \& Jewitt, D. 1995, AJ, 110, 794

Kolokolova, L., Hanner, M. S., Levasseur-Regourd, A.-C., \& Gustafson, B. Å. S. 2004, in Comets II, ed. M. C. Festou, H. U. Keller, \& H. A. Weaver (Tucson, AZ: Univ. Arizona Press), 577

Krivov, A. V., Löhne, T., \& Sremčević, M. 2006, A\&A, 455, 509

Lafrenière, D., Marois, C., Doyon, R., Nadeau, D., \& Artigau, É. 2007, ApJ, 660,770

Lagrange, A.-M., Bonnefoy, M., Chauvin, G., et al. 2010, Sci, 329, 57

Lagrange, A.-M., Gratadour, D., Chauvin, G., et al. 2009, A\&A, 493, 21

Lagrange, A.-M., Milli, J., Boccaletti, A., et al. 2012, A\&A, 546, A38
Marois, C., Lafrenière, D., Doyon, R., Macintosh, B., \& Nadeau, D. 2006, ApJ 641,556

Marois, C., Zuckerman, B., Konopacky, Q. M., Macintosh, B., \& Barman, T. 2010, Natur, 468, 1080

Milli, J., Mouillet, D., Lagrange, A.-M., et al. 2012, A\&A, 545, A111

Minowa, Y., Hayano, Y., Oya, S., et al. 2010, Proc. SPIE, 7736, 77363N

Pecaut, M. J., Mamajek, E. E., \& Bubar, E. J. 2012, ApJ, 746, 154

Roberge, A., Feldman, P. D., Weinberger, A. J., et al. 2006, Natur, 441, 724

Rodigas, T. J., Hinz, P. M., Leisenring, J., et al. 2012, ApJ, 752, 57

Song, I., Zuckerman, B., \& Bessell, M. S. 2012, AJ, 144, 8

Soummer, R., Pueyo, L., \& Larkin, J. 2012, ApJL, 755, 28

Strubbe, L. E., \& Chiang, E. I. 2006, ApJ, 648, 652

Suzuki, R., Kudo, T., Hashimoto, J., et al. 2010, Proc. SPIE, 7735, 773530

Tamura, M. 2009, in AIP Conf. Proc. 1158, Exoplanets and Disks: Their Formation and Diversity, ed. T. Usuda, M. Tamura, \& M. Ishii (Melville, NY: AIP), 11

Tanii, R., Itoh, Y., Kudo, T., et al. 2012, arXiv:1206.1215

Thalmann, C., Carson, J., Janson, M., et al. 2009, ApJL, 707, 123

Thalmann, C., Grady, C. A., Goto, M., et al. 2010, ApJL, 718, 87

Thalmann, C., Usuda, T., Kenworthy, M., et al. 2011, ApJL, 732, 34

Thébault, P., \& Augereau, J.-C. 2007, A\&A, 472, 169

Thébault, P., Kral, Q., \& Ertel, S. 2012, A\&A, 547, A92

van der Marel, R. P., Anderson, J., Cox, C., et al. 2007, Instrument Science Report ACS 2007-07, 22, 7

van Leeuwen, F. 2007, A\&A, 474, 653

Wyatt, M. C. 2008, ARA\&A, 46, 339 